\title{
NeW COMPUTER GaMe in MATLAB FOR EduCATional PURPoses
}

\author{
Karel Perutka \& Lenka Sarmanova
}
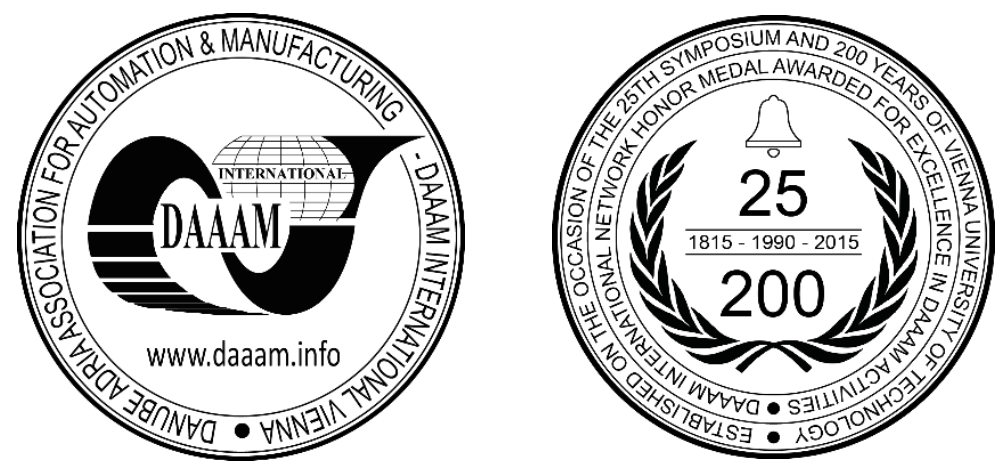

This Publication has to be referred as: Perutka, K[arel] \& Sarmanova, L[enka] (2017). New Computer Game in MATLAB for Educational Purposes, Proceedings of the 28th DAAAM International Symposium, pp.0070-0078, B. Katalinic (Ed.), Published by DAAAM International, ISBN 978-3-902734-11-2, ISSN 1726-9679, Vienna, Austria DOI: $10.2507 / 28$ th.daaam.proceedings.009

\begin{abstract}
This paper deals with the development environment for 2D computer game in the MATLAB. The theoretical part briefly describes the MATLAB environment and several games developed in this program, which are available on the MATLAB Central. The rest of the paper, the main part, is oriented practically, it contains the description of the game design. The game description is made in two versions, in a programmer and user perspective. The main part includes chapters about the game specification, game realization and the game from the user's point of view. The game is de facto original set of mazes and going through the maze is done after the right answer of question. The database of questions was created in purpose to practice the theoretical knowledge of MATLAB environment.
\end{abstract}

Keywords: MATLAB; GUI; Programming; Game

\section{Introduction}

Education in the sphere of scientific disciplines is a foundation in training of experts for high-tech industries. There are many tools and programs that have been developer and they can be used for educational purposes. It was followed by the usage of e-learning. After that, the educational tools were created. Teaching and practice using specific computer games became common during very last years.

Common education using lectures was presented for example by Goodwin. The lecture is an educational tool that allows a teacher to cover topic of a course that is to follow. It is a means of imparting knowledge by telling an audience (which can be of considerable size) a mixture of facts, opinions and interpretations [14]. Educational videos became the effective tool to improve training and education, for example in the medical area [17].

We can also mention some selected practical tool created in purpose to make the educational process much easier, such as graphical- based educational simulation tool called Gbest-WSN for simulating routing protocols of the static and mobile, homogeneous and heterogeneous WSNs [18]. Or, there was presented the tool that provides an easy and intuitive remote interaction with a team of mobile robots through Internet. It was designed with the aim that students of robotics carry out training with mobile robots from their own home, independently of the operating system they use and with a minimum installation in their computer [9]. RobotiCad is another example of such tool. It is a user-friendly Matlab/Simulink toolbox for the modeling and simulation of robotic manipulators [10]. There were also created the newly 
developed educational tools to encourage high school students' interest in STEM (Science, Technology, Engineering, Mathematics) disciplines. These tools include laboratory courses developed in the fields of physics, information technologies and mathematics. These activities were aimed at increasing the quality of STEM disciplines learning which resulted in higher quality of training of future engineers [11]. Next example of created educational tools is from the area of the artificial intelligence (AI). Artificial neural networks are some kind of data processing systems, which try to simulate features of the human brain and its learning process. So, they are widely used by researchers to solve different problems in optimization, classification, pattern recognition, associative memory and control. There was created the educational tool, which can be used to work on different kinds of neural network models and learn fundamentals of the artificial neural network [15]. Next mentioned example is from the area of uncertainty measurements. Uncertainty of measurements and parameters is frequently neglected by practitioners in the design of systems even in safety critical applications. Although there exists numerous tools to support uncertainty calculation, reasons for limited usage in early design phases may be low awareness of the existence of the tools and insufficient training in the practical application. Therefore, the teaching concept for uncertainty calculation in measurement science education was created. It is directly based on the utilization of software tools [16].

The games are the important aspect of the modern education. There was also suggested that online multiplayer educational games should be approached as a complex learning system, based on the principles of activity theory, where the Subjects would interact with other Subjects, Objects and Tools of the game, under specified Rules and create Communities through division of labor, leading to the expected learning outcome. Thus, there was suggest taking into account some important issues concerning the Subjects that the activity theory refers to, such as gender differences in playing games, academic performance, self-esteem and computer self-efficacy [12].

Augmented reality plays more important role in the educational process. The implementation of Mobile Pedestrian Navigation and Augmented Reality in mobile learning contexts shows new forms of interaction when students are taught by means of learning activities in formal settings. There was presented the educational, quantitative, and qualitative evaluation of an Augmented Reality and Mobile Pedestrian Navigation application. The software was designed for mobile learning in an educational context, to evaluate its effectiveness when applied as a teaching tool, in comparison to similar tools such as e-learning [13].

The name MATLAB originated from two English words, matrix and laboratory, which means "matrix and lab". MATLAB is the program environment that allows us to count a variety of mathematical operations, it can handle with vectors and matrices, plot charts, and create user interface applications. MATLAB is an advanced technical computing language and environment, which includes many tools to develop algorithms and perform data analysis and complex numeric computations. MATLAB has been chosen due to the high usage in education and research as well as in industry. There were formulated the following questions: Are existing tools suitable and do they include all topics we teach at our university? Is it necessary to create new teaching aid or game?

Based on the previous wide research in the area of modern teaching methods and tools, the necessity of new educational tool to practice MATLAB knowledge in attractive form appeared. The purpose of the study was to create supporting document for the practical realization. After that, the new computer game practicing the knowledge of MATLAB was created.

The paper is organized as it is followed. Firstly, the brief study of created MATLAB games was created. This is followed by the new game description. The advantages of created game are evaluated in the conclusion.

\section{Brief study of created games in MATLAB}

\subsection{Computer Games in MATLAB - MATLAB Central}

MATLAB Central is the main source of created projects that MATLAB users share. The games from the MATLAB Central servers are freely downloadable with permission of the authors. There are various categories of games such as logical, casual, shooting and 3D games.

This game had become very popular in recent years and is the expansion of 1024 with the same rules. User @bmtran transferred it to MATLAB and published it on MATLAB Central.

The game is controlled using the arrow keys on the keyboard. The goal of the game is to combine the same numbers together and reach the final 2048 value. Pressing the arrow on the keypad specifies the direction that all the tiles come out in the $4 \times 4$ field until they are stopped by another tile or edge.

Tiles of the same value are moved together and added together. At each turn or move in a field, a new tile with a minimum value of 2 appears, so if the player does not choose a good strategy, the whole field can be filled with tiles with different values that cannot be combined and the player cannot move in such a situation. The game is downloaded directly as an application, so its code cannot be seen. [1] 


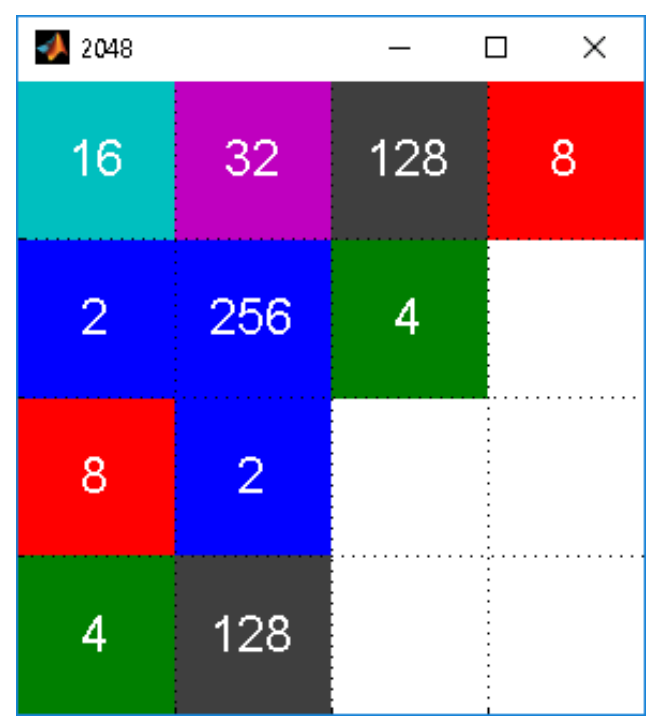

Fig. 1. 2048 game

Sudoku

The author of this famous MATLAB game is Stepen Sahrun. The goal of Sudoku is to fill in the empty spaces in the $9 \times 9$ field using numbers 1-9, so that in each row, column, and square the numbers 1-9 are not repeated. There is a choice of three difficulty options - light, medium or hard. There is also the option to reset the play - run it from the beginning, or run the solver to solve the game for us. Once the game has been completed, we have the option to run a game review to evaluate whether the field is filled correctly and, if not, to highlight the error boxes. Enter the numbers in each field using the numeric keypad. We use the mouse to select the game difficulty or reset, check, or running the solver.

The program contains two M-files, one for the game and the other for the sudoku solver. Together, both files have about 850 rows of code. [2]

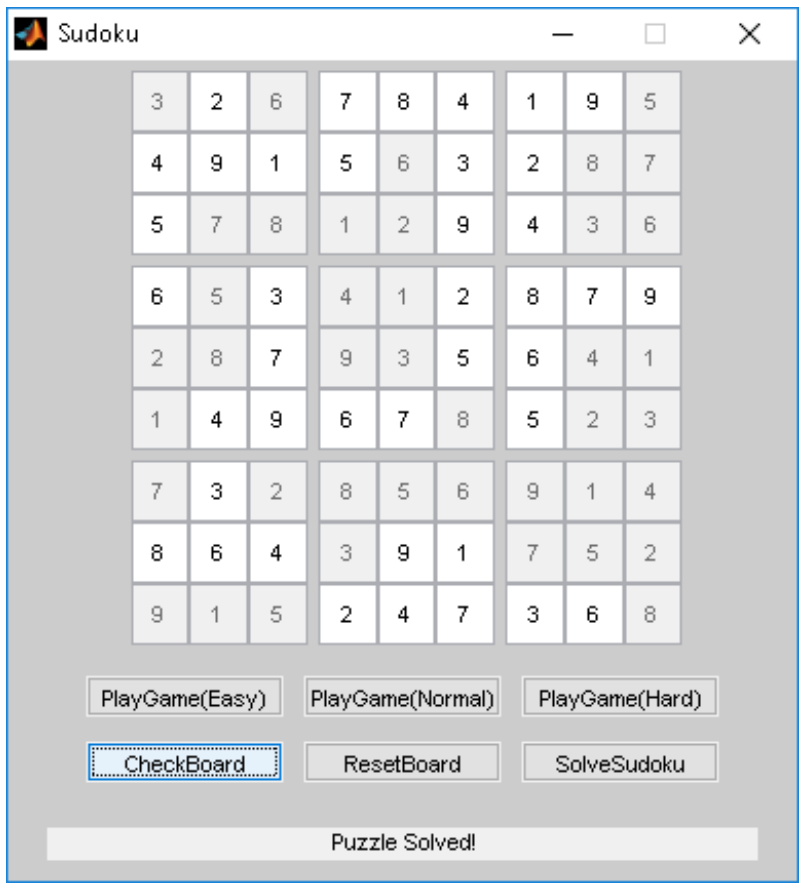

Fig. 2. Sudoku game

Matris

The Matris game is the similar game to the well-known Tetris game created by the MATLAB program, written by Mingjing Zhang. Matris is controlled using the mouse and keys. To start the game, click the Start button. The shapes fall randomly from the database, and the player's task is to fold the falling shapes side by side to fill the line and then disappear. 
With shapes, you can move left and right with the left and right arrows, use the up arrow to rotate the shape, and use the down arrow to speed it down to the ground. In the top right corner, a shape is displayed, which starts in the field as the next one, which allows the player to tact forward. In the right part of the window we also see information about the results of the game, namely our score and the number of disappeared rows.

With the increasing score of the game, the rate of crash of individual shapes increases, making the game more difficult. If a player fails to arrange shapes and some of the shapes touch the upper axis, the game ends. The game can also be paused at any time. The program contains one M-file with approximately 850 lines of code. The game has very nice graphics. [3]

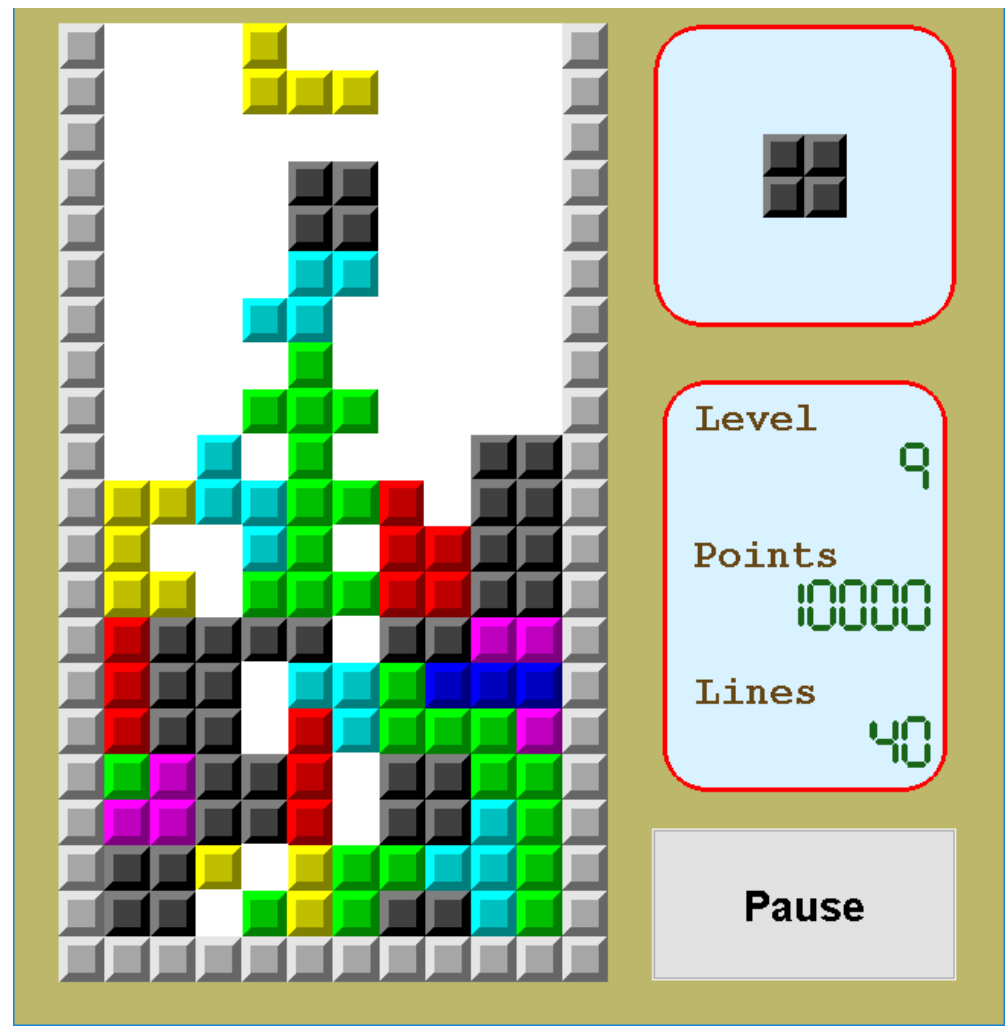

Fig. 3. Matris game

\subsection{Computer Games in MATLAB - Games created by students}

\section{Ludo}

The author of this classic board game is David Fiala, who created in 2013/2014 it as his thesis [6] on Mathematics 2D Computer Game.

The game is for 2-4 players, with players being either real people or a computer. The aim of the game is not to go through the player four pieces until the target field is determined by the same color as the player's pieces before the other players. Individual players alternate in a dice roll, the throw result indicating the number of shift boxes. If there is not yet one of our gaming figures in the field, but it's in the house, we have the option of $3 x$ throwing a dice until we hit number 6 , so we can put a piece from the house into the field.

If we have more than one piece in the field, we can select the number of pieces that will be moved by the number of squares given by the throw. If our figurine appears on a field where another player's figure is already in place, the opponent's figure must be returned to the house.

You can stop the game at any time, save it and run it again. We can also look into the rules during the game. The author has worked on creating the graphics of the game, so the game is graphically very good. In MATLAB CommandWindow, the game commentaries appear throughout the game, and on the right of the game window, we have an overview of the current state of the game, specifically which player is on the move. The program contains about $50 \mathrm{M}$-files, most of which are scripts by name, clearly defining functionality. 


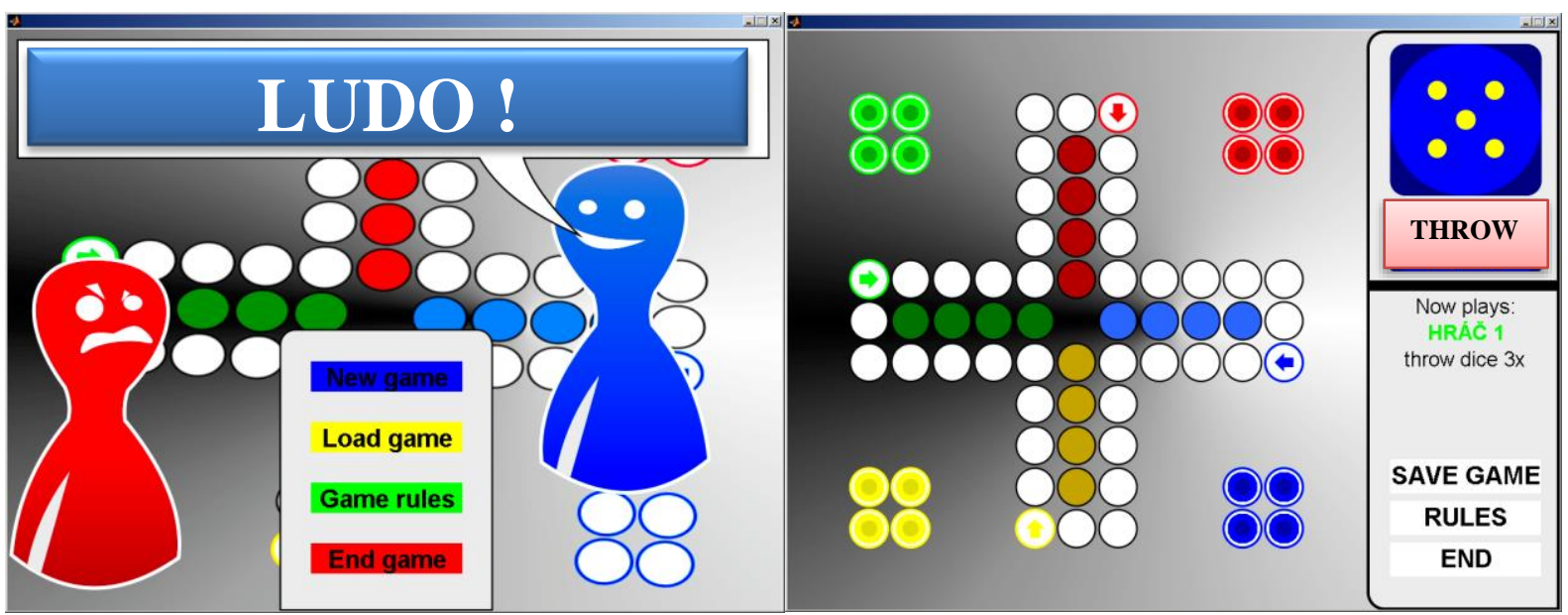

Fig. 4. Ludo game - first screen (left) and main dialog (right)

Risk

In 2005/2006, Michal Heczko, as a part of his work [5], he created the Tutorial and Testing Program for the PPAR, so called as Risk, and as the application aimed for MATLAB practicing.

The game is for 1-3 players. As the title suggests, the rules of the game are the same as the Risk television entertainment program. Specifically, in this game, the themes are focused on describing the MATLAB environment, operators, string operations, complex numbers, arrays, and matrices. The game is controlled by mouse and keyboard. The player on the move selects the topic and value of the question he / she wants to answer. If the answer is correct, it is added to the score. The game has a time limit for both the player's response and the lap length, both of which are represented by the progress bar. After the timeout, a player with more points wins.

The game itself contains about $40 \mathrm{M}$-files and 5 files with FIG. Since the entire app is a learning program, this game is de facto nice application for users who can verify their MATLAB knowledge in a funny way.

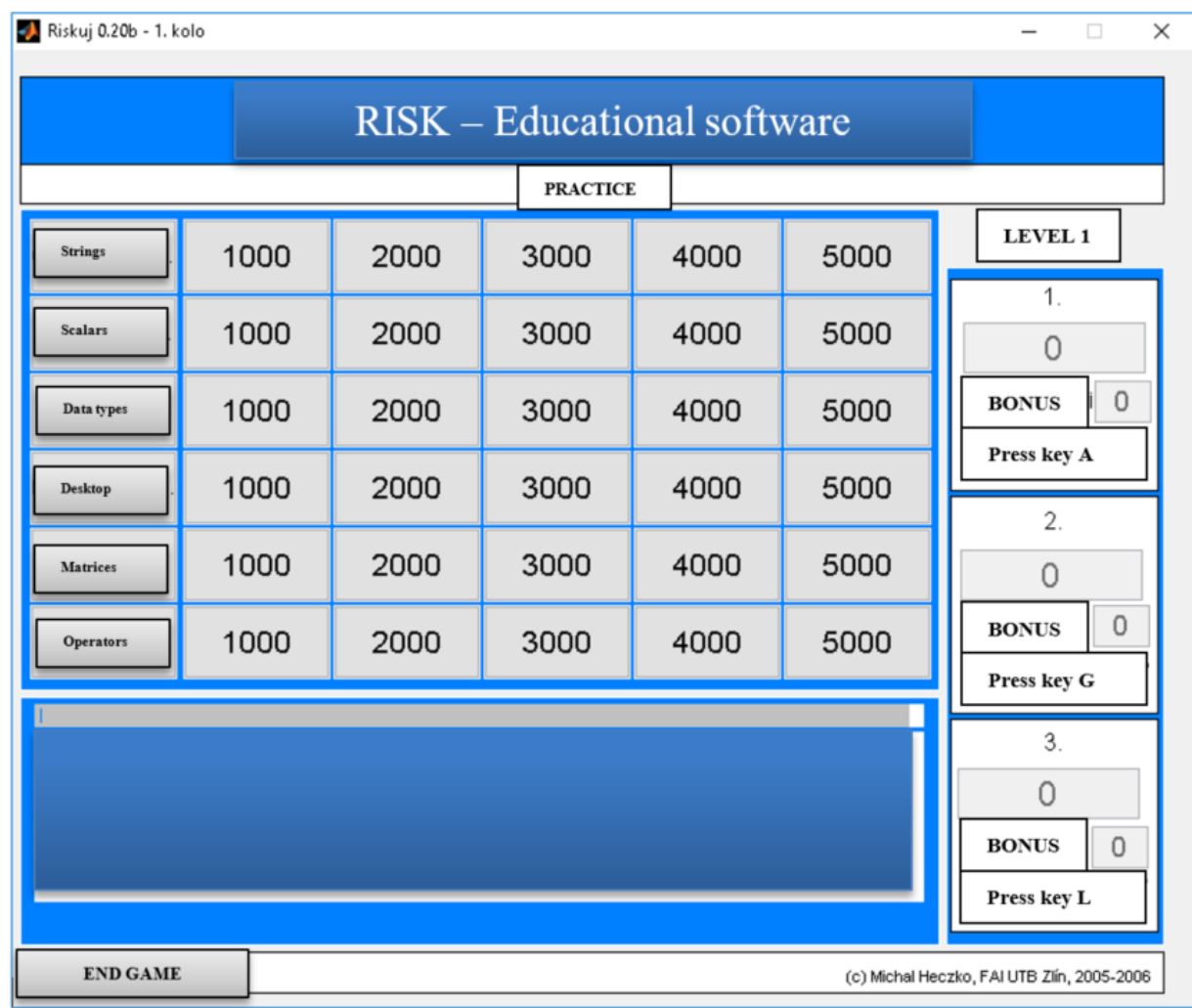

Fig. 5. Risk game

Other nice research papers were published [7], [8]. The results presented in these papers can be extended to educational purposes, too. 


\section{New Game Specification}

\subsection{Game description and parameters}

The new created game for educational purposes is named as Labyrinth of MATLAB. The game is for single player only [4]. The game contains a total of 10 rooms that the player has to pass within a given time limit. In each room, there is a labyrinth in which there are obstacles in the form of so-called gates. The player passes through the gateway if he correctly answers the question from the MATLAB usage.

The player's position in the labyrinth is indicated by a green square that you can move with the arrow keys. We answer the questions by entering a letter on the keyboard, such as a, b, c or d.

The Labyrinth of MATLAB can be played by anyone who is able to answer the questions correctly, it is necessary to have the basic experience with MATLAB. The game is based on the multiple activities at the same time when the player has to look for the right way to go through the labyrinth, keep track of time, collect as many points as possible and also show his/her knowledge of MATLAB.

You can run the game in the Command Window of MATLAB using the LabyrinthOfMatlab.m file, i.e. calling the LabyrinthOfMatlab command.

\subsection{Game rules}

The goal of the game is to go through ten rooms. In each room, there is a labyrinth that a player must pass through and reach the destination, or another room, within a two minute time limit.

In the labyrinth, obstacles in the form of so-called gates are placed during the journey to the goal, which the player removes only with the correct answers to questions about MATLAB. If a player responds badly or not at all, then the primary (yellow) gate will become a warning (orange) gate. In this situation, the player has only one attempt to get the right answer and the last option to remove the obstacle. If a player replies badly or not at all, a blinded wall becomes a barrier and the player blocks one of the possible paths to the target.

If a player answers the question correctly for the first time, he will not only remove the gambling but get 20 points for the correct answer, with the correct answer until the second try, the player gets 10 points. If a player walks through a room earlier than a given time limit, he earns 1 point for each remaining second.

The database has 200 questions. Each room has 20 questions, and labyrinths are designed to give the player more of his ability to reach the end, and he does not have to answer all the questions the room contains, at least at the first, simpler levels. In more sophisticated rooms, a player may block the wrong way to the finish and lose everything. The player has up to two attempts to destroy the gate with the correct answer. Questions are randomly generated at each game and their responses are always mixed, ensuring the uniqueness of each game.

The points earned after passing the room are counted and after the successful completion of the game, the player is entered into the table of the best players if the resulting score is among the top ten.

The game can be stopped, paused, or stored in memory and run at any time.

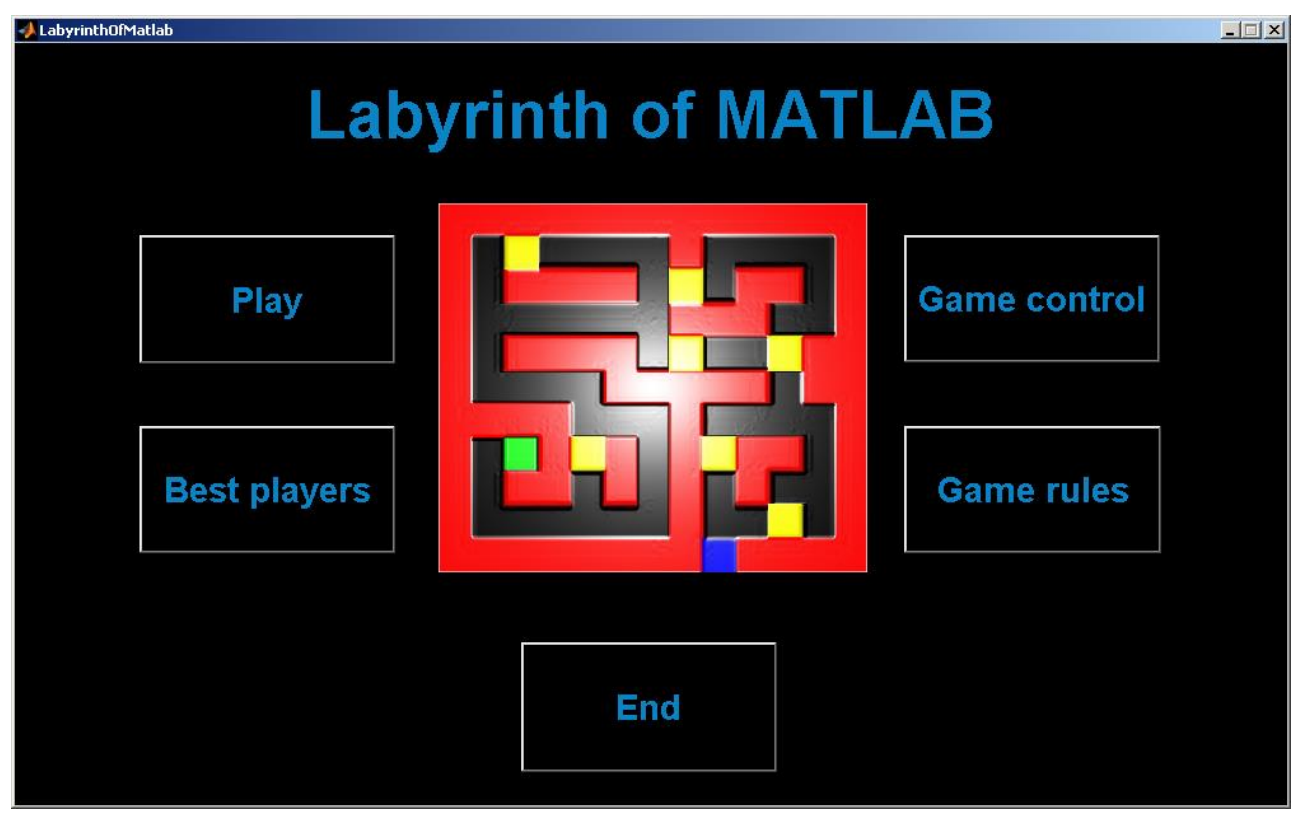

Fig. 6. Main screen of new game 


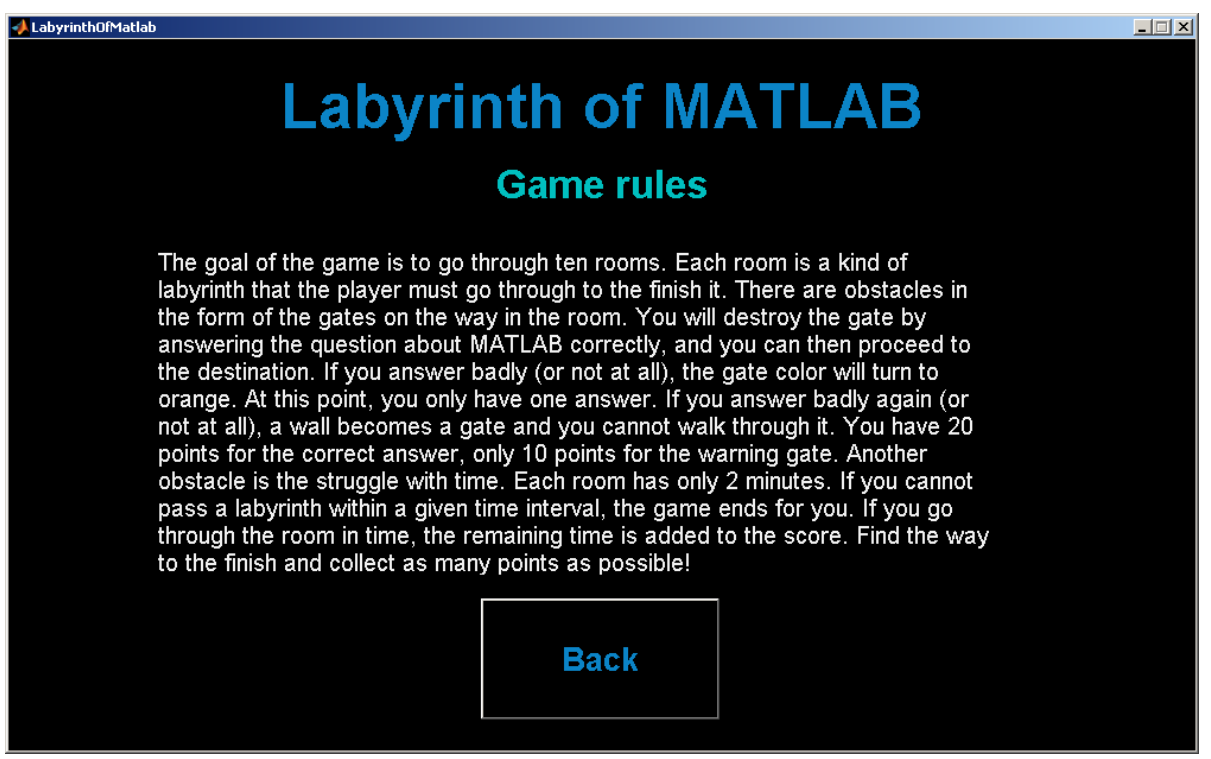

Fig. 7. Brief description of the rules directly in the game

\section{Game Realization}

\subsection{Game Description of game creation}

At first, we done the research about the MATLAB Central games and student papers, which are already described in the theoretical part of the thesis.

We then proposed the structure of the game. The game was developed in MALTAB version R2013a. We created two GUI files with which two main M-files are connected, and we added another eight M-files to make the program more transparent, so we did not repeat the source code.

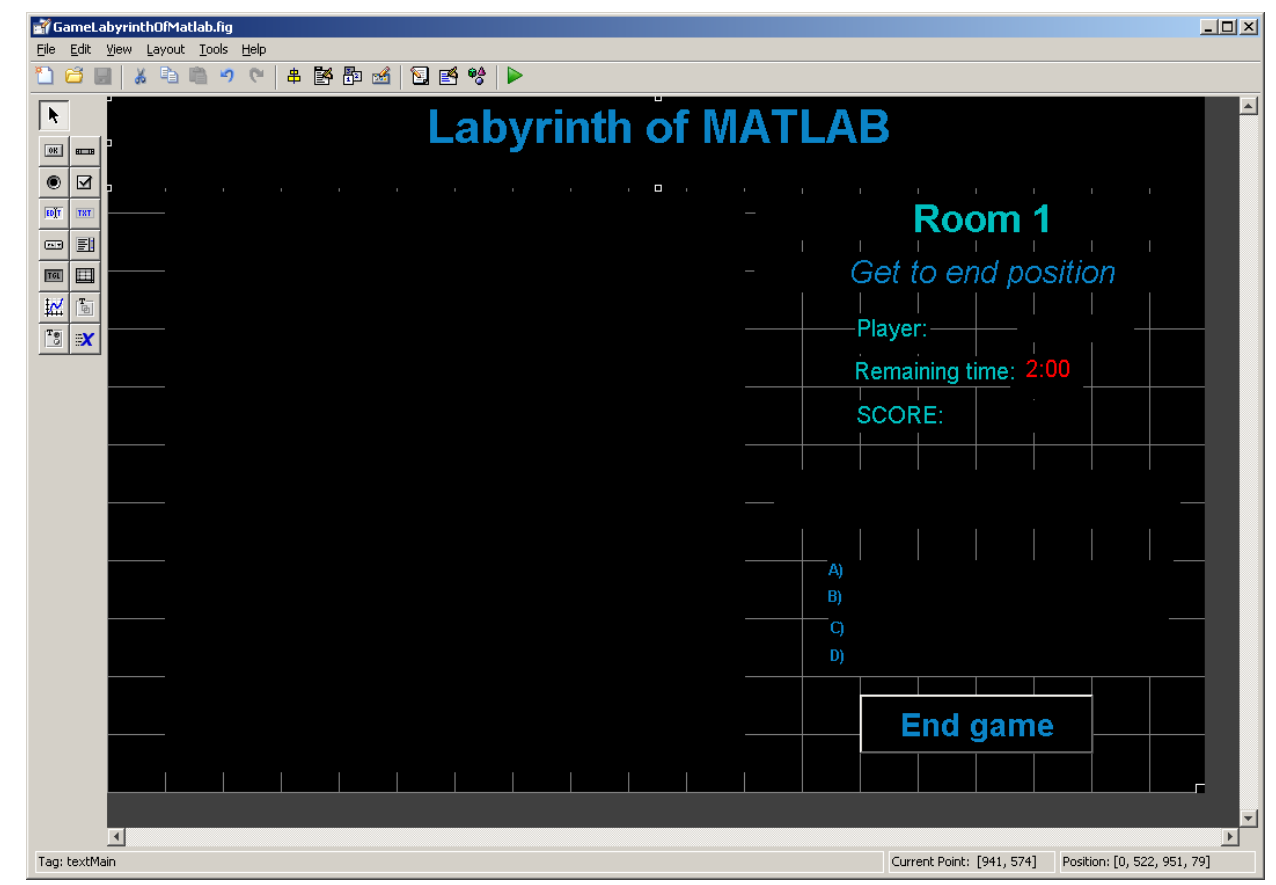

Fig. 8. Game GUI created using GUIDE

The main M-files LabyrinthOfMatlab.m and GameLabyrinthOfMatlab.m are linked so that when one ends, the second one starts and vice versa (except for the direct termination of the entire program in LabyrinthOfMatlab.m)

The structure of the main M-files is described by the following flow charts. 


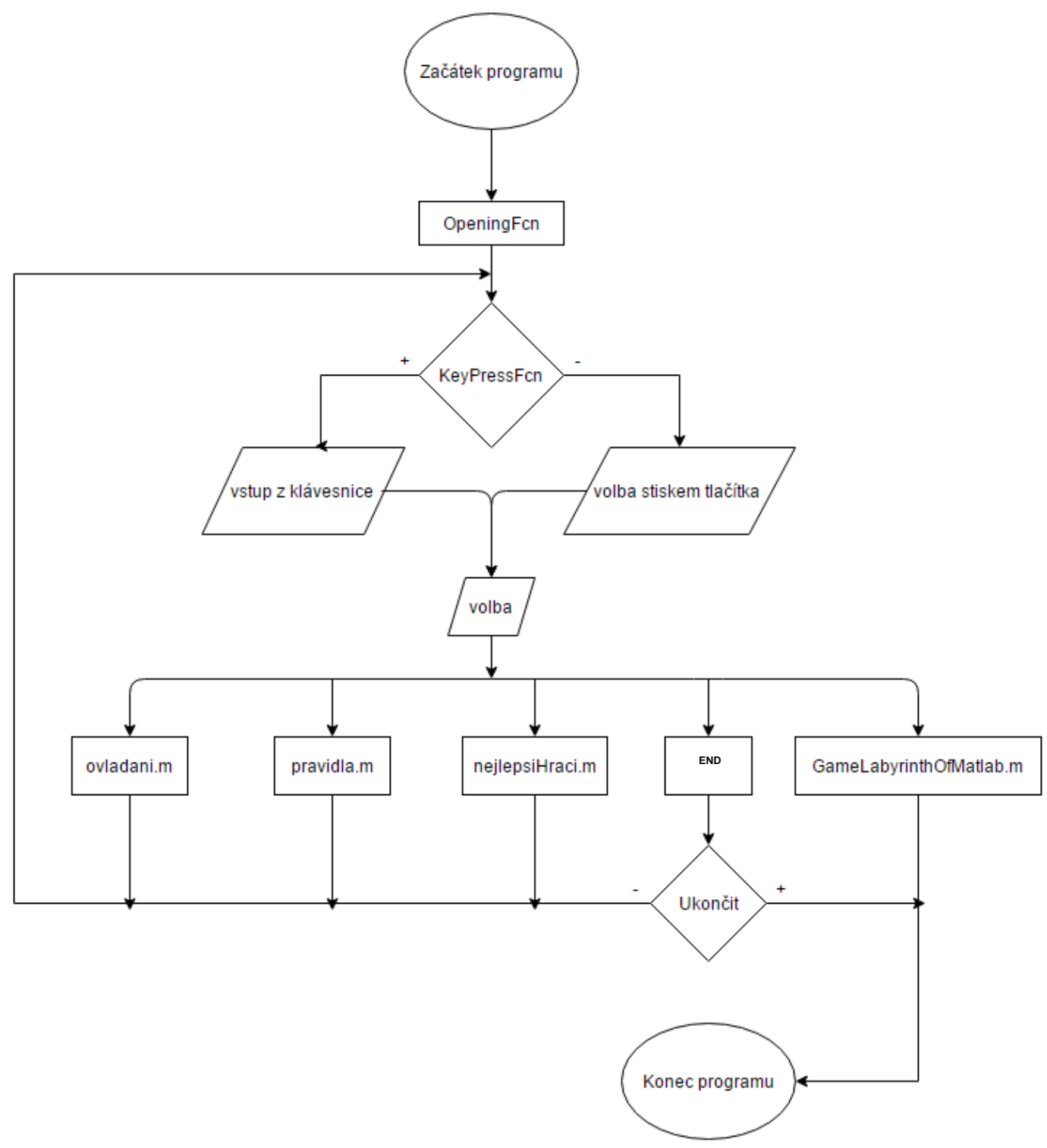

Fig. 9. Flowchart of LabyrinthOfMatlab.m file, the main file of the game

\subsection{Blocked path}

The game is programmed so that the user searches for the path to the labyrinth goal, so that he can evaluate whether to continue or not in the game when he / she blocks all the way to the target.

This problem was also a frequent reminder, although the game was designed in this way. However, this problem could easily be solved. The solution would be a function that keeps important gate positions for each room for each room while checking whether or not these gates are blocked. The following figure shows the solution.

In the following picture, you can see the first room in which an important gate is highlighted. The new function would therefore keep this gateway in line with its position and continuously monitor whether he pass or not. If a blocking has been registered, the game would alert the player that he no longer has the opportunity to reach the target. In other more difficult rooms, it would no longer matter just to one gate, as in the first room, but to more, which would not be a problem to control, but the algorithm for the other rooms would have been more complicated.

\section{Evaluation of created solution}

The created game became the part of new modern educational aids in according to the modern strategy of teaching that implements the lectures and practices in the attractive form. Games are leading applications in this area. The students confirm that they are more attract to studies. The tests that verified the MATLAB knowledge of students confirmed the better knowledge of students who played the game in comparison to those who did not use this game. 
The issue of the paper was to solve the problem - to find something to increase the knowledge of MATLAB programming at the faculty in the given curricula. It is important to understand the game functionality and to have the knowledge of MATLAB core to successfully finish the created game. The contents of the game is limited to MATLAB core only.

\section{Limitations}

Although the paper has reached its aims, there were some limitations. First, because the game is new, it was verified only by small group of students who was attending the classes. Therefore, it is necessary to use it for some years to generalize the result and to find what to improve and enlarge. Second, the content of the game is specific. It was created for the given curriculum, so it is necessary to modify or enlarge it in purpose to use this game by users from other faculty.

\section{Conclusion}

The paper presented newly created computer game in MATLAB that was created for practicing the knowledge of MATLAB programming in the attractive way. There was no specific tool practicing the lectures from the area of MATLAB programming at my faculty. It was the main problem. This problem was solved by new project, and lead to the creation of the new single player game in MATLAB. Before the problem solution, the research of existing games in MATLAB was done and this was followed by new game creation. This game was used during teaching lessons before final test and students like it. Future work will be focused on the comments of the testers and to enlarge the number of the rooms in purpose to practice more topics from MATLAB, not only the MATLAB core.

\section{References}

[1] 2048. MathWorks [online]. @bmtran, c1994-2017 [cit. 2017-09-29]. Available from: https://www.mathworks.com/matlabcentral/fileexchange/46028-2048

[2] Sudoku. MathWorks [online]. Stepen Sahrun, c1994-2017 [cit. 2017-09-29]. Available from: https://www.mathworks.com/matlabcentral/fileexchange/34988-sudoku

[3] MATRIS v0.10.10.09sb for Windows. MathWorks [online]. Mingjing Zhang, c1994-2017 [cit. 2017-09-29]. Available from: https://www.mathworks.com/matlabcentral/fileexchange/28985-matris-v0-10-10-09sb-forwindows

[4] Sarmanova, L. (2016). The Development of a Computer Game Environment in MATLAB, thesis, UTB in Zlin, Zlin.

[5] Heczko, M. (2006). Tutorial and testing software for the subject "Computer aid of automatic control", thesis, UTB in Zlin, Zlin.

[6] Fiala, D. (2014). A 2D Computer Game in the MATLAB Environment, thesis, UTB in Zlin, Zlin.

[7] Haskovic, D., Katalinic, B. \& Kukushkin I. (2015). Role of the Adviser Module in the Hybrid Assembly Subordinating Control Structure, Procedia Engineering, Volume 100, ISSN 1877-7058, pp. 1706-1713.

[8] Gastermann, B., Stopper, M., Kossik, A. \& Katalinic, B. (2015). Secure Implementation of an On-premises Cloud Storage Service for Small and Medium-sized Enterprises, Procedia Engineering, Volume 100, ISSN 1877-7058, pp. 574-583.

[9] Payá, L., Reinoso, O., Sánchez, A., Gil, A. \& Fernandéz, L. (2010). An educational tool for mobile robots remote interaction, IFAC Proceedings Volume, Volume 42, Issue 24, ISSN 1474-6670, pp. 180-185.

[10] Falconi, R. \& Melchiorri, C. (2008). RobotiCad: an Educational Tool for Robotics, IFAC Proceedings Volume, Volume 41, Issue 2, ISSN 1474-6670, pp. 9111-9116.

[11] Mayorova, V., Grishko, D. \& Leonov, V. (2017). New educational tools to encourage high-school students' activity in stem, Advances in Space Research, In press, ISSN 0273-1177.

[12] Paraskeva, F., Mysirlaki, S. \& Papagianni, A. (2010). Multiplayer online games as educational tools: Facing new challenges in learning, Computers \& Education, Volume 54, Issue 2, ISSN 0360-1315, pp. 498-505.

[13] Joo-Nagata, J., Martinez Abad, M., García-Bermejo Giner, J. \& García-Peñalvo, F.J,. (2017). Augmented reality and pedestrian navigation through its implementation in m-learning and e-learning: Evaluation of an educational program in Chile, Computers \& Education, Volume 111, ISSN 0360-1315, pp. 1-17.

[14] Goodwin, A.P.L. (2003). The lecture as an educational tool-a personal view, Current Anaesthesia \& Critical Care, Volume 14, Issue 4, ISSN 0953-7112, pp. 170-172.

[15] Deperlioglu, O. \& Kose, U. (2011). An educational tool for artificial neural networks, Computers \& Electrical Engineering, Volume 37, Issue 3, ISSN 0045-7906, pp. 392-402.

[16] Zangl, H. \& Hoermaier, K. (2017). Educational aspects of uncertainty calculation with software tools, Measurement, Volume 101, ISSN 0263-2241, pp. 257-264.

[17] Phillips, J., Brook, A., Tseng, I., Sharpe, R.E., Fein-Zachary, V., Slanetz, P.J. \& Mehta, T.S. (2016). Educational Videos: An Effective Tool to Improve Training in Interventional Breast Procedures, Journal of the American College of Radiology, Volume 13, Issue 6, ISSN 1546-1440, pp. 719-724.

[18] Sabor, N., Sasaki, S., Abo-Zahhad, M. \& Ahmed, S.M. (2016). A Graphical-based educational simulation tool for Wireless Sensor Networks, Simulation Modelling Practice and Theory, Volume 69, ISSN 1569-190X, pp. 55-79. 